\title{
Correlación entre la estructura de bandas y las propiedades físicas de óxidos cerámicos de estructura perovsquita con metales de transición (II): Propiedades magnéticas y electrocatalíticas.
}

\author{
L. SÁNCHEZ, J.R. JURADO \\ Instituto de Cerámica y Vidrio, CSIC, 28500 Arganda del Rey, Madrid.
}

\begin{abstract}
Se revisan los principales modelos teóricos y parámetros presentes en la literatura, que pretenden dar una explicación sobre las propiedades magnéticas y electrocatalíticas de óxidos cerámicos con estructura perovsquita, en los que en la posición $\mathrm{B}$ se encuentran los metales de transición ( $\mathrm{Fe}, \mathrm{Co}, \mathrm{Ni}, \mathrm{Mn}$ ), en relación con su estructura de bandas y el carácter de sus electrones más externos. Se pretende integrar y dar una visión global clara a dichos modelos inconexos.
\end{abstract}

Palabras clave: óxidos cerámicos de tipo perovsquita, magnetismo, catálisis, estructura de bandas.

Relationship between band structure and Physical properties in transition metal ceramic oxides with perovskite structure: (II) Magnetic and electrocatalytic properties.

Theoretical models present in literature and developed for the explanation of magnetic and electrocatalytic properties in transition metal ( $\mathrm{Fe}, \mathrm{Co}, \mathrm{Ni}, \mathrm{Mn})$ ceramic oxides with perovskite structure in relation with their band diagram and outer electron nature have been revised. It is proposed to give an integrated and global idea about of several theoretical models that have not been yet connected.

Key words: ceramic oxides with perovskite structure, magnetism, catalysis, band structure.

\section{INTRODUCCIÓN}

El objetivo del presente trabajo es la revisión de los modelos presentes en la literatura sobre orden y comportamiento magnético, en los óxidos cerámicos de estructura perovsquita, que contienen metales de transición en su composición, en relación con la naturaleza de sus electrones más externos y el modelo de bandas $(1,2)$ revisado en la primera parte de este trabajo. De forma similar se tratan las características electrocatalíticas que son especialmente interesantes en estos óxidos por sus aplicaciones en dispositivos electroquímicos. Tanto en el presente trabajo de revisión como en su parte previa se pretende la integración de los modelos teóricos para la comprensión global de todas las propiedades físicas de los compuestos citados.

\section{PROPIEDADES MAGNÉTICAS. MAGNETORRESISTENCIA.}

El estudio sistemático de las propiedades de los electrones d en estas perovsquitas (carácter colectivo a través de la teoría de bandas convencional, o localizado a través de la teoría de campo cristalino, junto al supercanje y/o doble canje) desarrollado por Goodenough y colaboradores $(2,3)$, permite la comprensión de los distintos comportamientos magnéticos. Además esta estructura cristalina facilita dicho objetivo, al minimizar las interacciones magnéticas competitivas entre los momentos de cationes vecinos. Así se asegura que experi- mentalmente se pueden comprobar las reglas semiempíricas, que se citarán a continuación, acerca de las interacciones de supercanje, doble canje, y las predicciones de ordenamiento magnético y momentos magnéticos espontáneos empleando parámetros como la energía de transferencia $b(1,2)$ entre vecinos más próximos, así como la temperatura y el número de electrones $n_{1}$ más externos por cada orbital, para el estudio del carácter de los electrones y los procesos anteriormente expuestos.

El magnetismo espontáneo de electrones colectivos, sucede solamente en un estrecho intervalo de b, que corresponde a una zona intermedia entre estados magnéticos de electrones localizados y paramagnetismo de Pauli de electrones colectivos.

Los fenómenos magnetorresistivos exigen tener en cuenta otros factores fundamentales, como las interacciones intensas entre electrones, y el acoplamiento electrón-fonón no incluidos en el tratamiento anterior.

\subsection{Teoría de bandas. Electrones colectivos.}

En la teoría de bandas convencional la descripción de los electrones más externos se construye a partir de funciones de Bloch, y sus energías (4). Podemos incluir dos modelos de comportamiento magnético de electrones colectivos que además se observan en estos óxidos. 


\section{1.1. FERROMAGNETISMO DE STONER}

Se trata de un modelo fenomenológico (5) que exige que los electrones responsables del ferromagnetismo se sitúen en bandas parcialmente llenas de modo que existan niveles energéticos vacantes para permitir, que los electrones con spines desapareados se muevan en ellas, y aparezca una imanación espontánea. La distancia entre átomos debe ser adecuada, para que las fuerzas de canje puedan alinear los spines de electrones de átomos vecinos.

El campo molecular descompensa las energías de los electrones, con distinto spin y el equilibrio del sistema exige el giro de spin de algunos electrones obligando a que se sitúen en niveles superiores de la banda. Para que la fuerza de canje (generada por el campo molecular de Weiss), sea capaz de producir estos saltos en la banda, los niveles energéticos de ésta han de estar muy cercanos (D(E) alta, y de banda estrecha). En ciertas condiciones de temperatura o de transiciones de densidad electrónica, puede darse la rotación de estos spines mediante una onda de densidad de spines $\operatorname{SDW}(6,7)$, como puede verse en el $\mathrm{Pb}_{1-x} \mathrm{Sr}_{x} \mathrm{RuO}_{3}$ (2).

\section{1. 2. PARAMAGNETISMO DE PAULI}

Básicamente los principios que rigen este fenómeno son similares a los del anterior (5), salvo que, en este caso no aparece el campo molecular interno, sino que se aplica un campo externo cuyo efecto, a $0 \mathrm{~K}$, es girar los spines de los electrones que están cercanos a $\mathrm{E}_{\mathrm{F}}$. Sólo para ellos el campo tiene la suficiente fuerza como para ponerlos paralelos a sí mismos, y reducir así la energía del sistema. El resultado es un paramagnetismo débil. Debe tenerse en cuenta que en este caso el campo aplicado crea el momento en cada átomo, en contraste con lo que sucede en el paramagnetismo normal. El incremento de la temperatura solamente excita los electrones de la parte superior de la banda a niveles superiores, pero su efecto es idéntico en los estados de spin sin interferir en el efecto del campo, por ello en este caso la susceptibilidad es independiente de la temperatura. Casos de este tipo son el $\mathrm{CaTiO}_{3}$ y el $\mathrm{SrTiO}_{3}$

La diferencia fundamental entre el paramagnetismo de Pauli y el ferromagnetismo de Stoner está en la fuerza que genera la descompensación de los spines, en el primer caso es un campo magnético aplicado y en el segundo un campo molecular de Weiss (5) que genera las fuerzas de canje de forma espontánea y permanente.

\section{2. Orden magnético. Electrones localizados.}

Veamos previamente un estudio introductorio de los términos magnéticos o de spin que forman parte del Hamiltoniano que describe los orbitales de estados localizados y sus energías $(1,2)$ :

$$
H=H_{0}+V_{\text {int }}+\mathrm{V}_{\mathrm{cr}}+\left(V_{L S}+V_{\lambda}+H_{Z}+\Sigma_{j} V_{i j}\right)
$$

Los términos que interesan en esta ocasión son $\left(V_{L S}+V_{\lambda}+H_{Z}+\Sigma_{j} V_{i j}\right)$ y no pueden ser tratados por separado, ni aislados de la aportación no cúbica del campo cristalino al potencial. Siguiendo la clasificación de Goodenough y Longo (3) podemos estudiarlos como sigue:

1. $V_{L S}$ es el potencial debido a la interacción spin-órbita, que genera los multipletes asociados a un determinado $\mathbf{J}$ a través de la interacción entre $\mathbf{L}$ y $\mathbf{S}$ :

$$
V_{L S}=\lambda \mathbf{L}\langle\mathbf{S}=\lambda / 2[\mathrm{~J}(\mathrm{~J}+1)-\mathrm{L}(\mathrm{L}+1)-\mathrm{S}(\mathrm{S}+1)]
$$

Donde el valor absoluto del parámetro $\lambda$ es del orden de 100 a $1000 \mathrm{~cm}^{-1}$ en el caso de metales de transición del grupo del Fe, Co y Ni. (8). El acoplo spin órbita introduce una simetría axial en la distribución de carga, donde el spin (o el momento atómico) define el eje. La anisotropía cristalina y magnética convergen aquí.

2. $V_{l}$ se refiere a una interacción de muchos átomos y representa la energía de acoplo elástico entre posiciones catiónicas, que presentan una distribución de inferior simetría, que la cúbica como consecuencia de una ordenación de los electrones en los orbitales localizados.

3. $H_{Z}$ es la energía de Zeeman debida a las interacciones de canje interatómico, que generan un campo molecular interno cuya consecuencia es la aparición de un desplazamiento en los niveles de orbitales con distinto spin.

4. $V_{i j}$ se refiere a la interacción spin-spin interatómica y se relaciona con las integrales de canje $\mathbf{J}_{\mathrm{ij}}$ (5) y esta a su vez con la integral de transferencia $(2,3)$. El orden magnético a largo alcance de los momentos atómicos en el caso de electrones localizados en un cristal, sucede a temperaturas inferiores a un valor crítico debido a este acoplo spin-spin entre átomos vecinos. $Y$ contiene los términos referidos al canje directo, al doble canje (presente sólo en el caso de existir cargas itinerantes), y a los distintos tipos de supercanje. Todas las interacciones entre spines dependen de la relación entre energías coulombianas y el principio de exclusión de Pauli.

5. El estudio del ordenamiento magnético en perovsquitas, está siendo fundamental en el desarrollo de modelos microscópicos para la comprensión de los fenómenos que se han citado; básicamente para establecer los signos y la magnitud de las $\mathrm{J}_{\mathrm{ij}}$ en diversos casos. Esto se debe a que sólo las interacciones entre catión-anión-catión en torno a $180^{\circ}$ son posibles en esta estructura al ser únicamente los cationes A o B magnéticos, de este modo se eliminan las complicaciones presentes cuando aparecen interacciones competitivas.

a) Canje directo

Entre electrones que interaccionan en átomos vecinos en un cristal, se reducen las fuerzas repulsivas haciendo aparecer un canje directo de carácter ferromagnético $\left(\mathrm{J}_{\mathrm{ij}}^{\mathrm{D}}>0\right)(8)$. En el caso de electrones localizados esta interacción es más débil que las de supercanje $\mathrm{J}_{\mathrm{ij}}^{\mathrm{s}}$

b) Supercanje

Dado que los cationes magnéticos de estos compuestos están separados por aniones oxígeno no magnéticos, las interacciones spin-spin estarán mediatizadas por los electrones de estos aniones, de ahí el fenómeno de supercanje. La peculiar estructura de los orbitales cristalinos en el caso de electrones localizados $\varphi_{\mathrm{e}}$ y $\varphi_{\mathrm{t}}(6)$, en los que se mezclan estados excitados para generar otros de menor energía, son los que permiten la aparición de este supercanje.

El tipo de interacción habitual en la mayoría de perovsquitas $\mathrm{ABO}_{3}$ se debe a la presencia de supercanje entre cationes idénticos con orbitales semillenos generando así una interacción antiferromagnética para los átomos $\mathrm{B}$. La magnitud de esta interacción antiferromagnética(3) es:

$$
4 S^{2} \mathrm{~J}_{\mathrm{ij}}^{\mathrm{S}}=-2 \mathrm{~b}_{\mathrm{ij}}^{2} / \mathrm{U}
$$

siendo $b_{\mathrm{ij}}$ la integral de transferencia y U la energía electrostática necesaria para añadir un electrón a un orbital semilleno. Aún así existen otros casos específicos, en que transiciones de tipo Janh-Teller (2) pueden inducir una anisotropía en la interacción magnética y modificar el tipo de acoplo (caso de la $\mathrm{LaMnO}_{3}$ ). 
Cuando los cationes B están en distinto estado de valencia, el signo de las interacciones de supercanje entre catiónanión-catión depende notablemente de los cambios en el ángulo de solapamiento, ya que las variaciones frente al caso ideal de $180^{\circ}$ pueden generar el solapamiento entre orbitales $\mathrm{t}_{2 \mathrm{~g}}$ de un catión y $\mathrm{e}_{\mathrm{g}}$ del otro. En los óxidos cuando el ángulo es superior a $145^{\circ}$ no es necesario tener en cuenta este efecto $(3,9)$

Las anisotropías cristalinas asociadas con los octaedros deformados de la estructura perovsquita, pueden llegar a inclinar los spines de una red antiferromagnética, para generar un ferromagnetismo débil o parasitario ( $\mathrm{LaFeO}_{3^{\prime}}$ $\mathrm{SmFeO}_{r}, \mathrm{LaMnO}_{3}$ ), que consiste en la aparición de una dependencia de la imanación : $\sigma=\sigma_{\mathrm{o}}+\chi \mathrm{H}$ (Ec.8) a altos campos ( $\mathrm{H}>6000 \mathrm{Oe}$ ), donde la $\sigma_{\mathrm{o}}$ es la imanación parasitaria y $\chi$ es la susceptibilidad específica. Este efecto es importante en los interacciones A-A y A-B presentes en ferratos y cromatos con estructura ortorrómbica y que contienen tierras raras en su composición pero puede ser reducido por un estricto control de la estequiometría del sistema (10).

c) Doble canje

Sólo existe si aparece un número no entero de electrones d por catión B. En el caso de una estequiometría perfecta es precisa la presencia de defectos. Para valores pequeños del número de cargas móviles por átomo $\mathrm{B}$ y bandas estrechas (1), surge en este caso, una interacción ferromagnética de doble canje(8).

En ocasiones la suma de los efectos de supercanje y doble canje, logra que la configuración más favorable sea una espiral antiferromagnética de spines $\left(\mathrm{SrFeO}_{3}\right)$ con ángulo de inclinación $\theta_{\alpha}$ (3) calculable, y que depende de la integral de transferencia $b^{\mathrm{D}}{ }_{\mathrm{ij}} \mathrm{y}$ de la de canje $\mathrm{J}_{\mathrm{ij}}$. Así mismo aparece un valor crítico del número de cargas móviles (2), por encima del cual la componente neta ferromagnética presente en dicha espiral se hace preponderante, y se llega al orden ferromagnético puro $\left(\theta_{\alpha}=0\right)$.

Goodenough y Longo (3) proponen para los distintos estados magnéticos de las perovsquitas unos diagramas de fases, en función de diversos parámetros como b, $\mathrm{n}_{1} \mathrm{y}$ la temperatura, y del carácter localizado o colectivo de los electrones, que forman parte de estas estructuras. De esta manera estos autores establecen un paralelismo entre el comportamiento magnético, y las bandas de energía de estos compuestos, a continuación veremos algunos comportamientos, que sin embargo estos criterios no engloban y también extenderemos el modelo a los procesos catalíticos relacionados tanto con la conducción electrónica como con el orden magnético.

La tabla I recoge los datos magnéticos de algunas perovsquitas.

Lista de abreviaturas: $\mathrm{n}_{\mathrm{A},}, \mathrm{n}_{||}^{\mathrm{A}}$ - Momento atómico o su componente paralela al momento ferromagnético neto en número de magnetones de Bohr: $\mathrm{p}_{\mathrm{A}}=\mathrm{n}_{\mathrm{A}} \mu_{\mathrm{B}}$.

$n_{e f}$ - Momento paramagnético efectivo: $p_{e f}=n_{e f} \mu_{B}$.

$\mathrm{T}_{\mathrm{C}}[\mathrm{K}]$ - Temperatura de Curie ferro y ferri magnética.

$\mathrm{T}_{\mathrm{N}}[\mathrm{K}]$ - Temperatura de Nèel, antiferromagnética.

$\mathrm{T}_{\mathrm{r}}[\mathrm{K}]$ - Temperatura de reorientación de spines.

$\mathrm{T}_{\mathrm{p}}[\mathrm{K}]$ - Temperatura de Curie paramagnética, $\mathrm{T}_{\mathrm{p}<0}$ si hay acoplo antiferromagnético.

$\sigma_{0}[\mathrm{emu} / \mathrm{g}]$ - imanación parasitaria efectiva: $\sigma=\sigma_{0}+\chi_{\mathrm{g}} \mathrm{H}$. $\chi_{\mathrm{g}}[\mathrm{emu} / \mathrm{g}]$ - susceptibilidad paramagnética específica.
TABla1. Propiedades MAgNÉTICAS DE ÓXIDOS CON ESTRUCTURA PEROVSQUITA Y METAL DE TRANSICIÓN.

\begin{tabular}{|c|c|c|}
\hline Compuesto & Simetría (3) & Propiedades magnéticas $(3,28)$ \\
\hline $\mathrm{LaFeO}_{3}$ & Ortorrómbica & $\begin{array}{l}\text { Antiferromagnetismo (fig. 1), } \mathrm{T}_{\mathrm{N}}=750 \mathrm{~K} \text {, } \\
\mathrm{n}_{\mathrm{Fe}}(4.2 \mathrm{~K})=4.65 \text {, Ferromagnetismo parasitario: } \sigma_{0} \| \mathrm{c}, \\
\mathrm{n}^{\mathrm{Fe}} \|=0.047, \mathrm{~T}_{\mathrm{C}}=743^{\circ} \mathrm{K}\end{array}$ \\
\hline $\mathrm{SmFeO}_{3}$ & Ortorrómbica & $\begin{array}{l}\text { Susceptibilidad paramagnética } \mathrm{T}=77^{\circ} \mathrm{K}, 300^{\circ} \mathrm{K} \text {. } \\
\text { Ferromagnetismo parasitario: } \sigma_{0} \| \mathrm{c} \text { si } \mathrm{T}_{\mathrm{r}}<\mathrm{T}<\mathrm{T}_{\mathrm{C}} \text { con } \\
\mathrm{T}_{\mathrm{C}}=675^{\circ} \mathrm{K} \text { y } \quad 440<\mathrm{T}_{\mathrm{r}}<460{ }^{\circ} \mathrm{K}, \sigma_{0} \| \text { a si } \\
77<\mathrm{T}<\mathrm{T}_{\mathrm{r}}, \sigma_{0}=0 \text { si } \mathrm{T}=4.2^{\circ} \mathrm{K}, \mathrm{n}_{\mathrm{Fe}}=0.041 \\
\text { Magnetostricción. }\end{array}$ \\
\hline $\mathrm{YFeO}_{3}$ & Ortorrómbica & $\begin{array}{l}\text { Antiferromagnetismo (fig. 1), } \mathrm{T}_{\mathrm{N}}=645^{\circ} \mathrm{K} \text {. } \\
\text { Ferromagnetismo parasitario: } \sigma_{0} \| \mathrm{c}, \mathrm{n}_{\mathrm{Fe}}=0.045 \text {. } \\
\text { Supercanje antisimétrico responsable de la inclinación } \\
\text { de los spines. } \\
\mathrm{T}_{\mathrm{p}}=-1400^{\circ} \mathrm{K} \text {. }\end{array}$ \\
\hline $\mathrm{SrFeO}_{3}$ & Cúbica & $\begin{array}{l}\text { Antiferromagnetismo tipo helicoidal, } \mathrm{T}_{\mathrm{N}}=134^{\circ} \mathrm{K}, \\
\mathrm{n}_{\mathrm{Fe}}(77 \mathrm{~K})=2.7,77<\mathrm{T}<500 \text { marcada desviación de la } \\
\text { Ley de Curie Weiss. }\end{array}$ \\
\hline $\mathrm{CaFeO}_{3}$ & Cúbica & $\begin{array}{l}100<\mathrm{T}<300{ }^{\circ} \mathrm{K} \text { Antiferromagnetismo (fig. } 2 \text { ), } \mathrm{T}_{\mathrm{N}}=120 \\
{ }^{0} \mathrm{~K}, \mathrm{n}_{\mathrm{ef}}=2.20 \text {, estado } \mathrm{Fe}^{4+}, \mathrm{T}_{\mathrm{p}}=-78^{\circ} \mathrm{K} \text {. }\end{array}$ \\
\hline $\mathrm{LaCoO}_{3}$ & $\mathrm{R} 3 \mathrm{c} \leftrightarrow \mathrm{R} 3 \leftrightarrow \mathrm{R} 3 \mathrm{~m}$ & $\begin{array}{l}\text { Aparece una fase ferro o ferrimagnética dispersa en } \\
\text { una matriz no magnética, este efecto es menos } \\
\text { pronunciado en policristales y genera curvas de } \\
\text { histéresis no lineales. } \\
0<\mathrm{T}<400^{\circ} \mathrm{K} \text { Minoria de iones cobalto en estado de alto } \\
\text { spin }\left(\mathrm{Co}^{3+}\right) \text { distribuidos al azar entre los de bajo spin. } \\
400<\mathrm{T}<650^{\circ} \mathrm{K} \text { ordenamiento de los iones de alto y } \\
\text { bajo spin en planos (111) alternativamente. }\end{array}$ \\
\hline $\mathrm{YCoO}_{3}$ & Ortorrómbica & $\begin{array}{l}300<\mathrm{T}<1300^{\circ} \mathrm{K} \text {, la susceptibilidad crece con la } \\
\text { temperatura entre } 500 \text { y } 800^{\circ} \mathrm{C} \text {. } \\
\mathrm{Co}^{3+} \text { diamagnético a bajas } \mathrm{T} \text {. }\end{array}$ \\
\hline $\mathrm{SrCoO}_{3-\mathrm{x}}$ & & $\begin{array}{l}\text { Fase "Brownmillerite"- Antiferromagnética, } \mathrm{T}_{\mathrm{N}}=570 \\
{ }^{\circ} \mathrm{K} \text {, aislante. } \\
\text { Fase Hexagonal- Paramagnética si } \mathrm{T}>77^{\circ} \mathrm{K} \text {, buen } \\
\text { conductor. } \\
\text { Otras fases- Ferromagnética, } \mathrm{T}_{\mathrm{C}}=160-300^{\circ} \mathrm{K} \text {, buen } \\
\text { conductor. }\end{array}$ \\
\hline $\mathrm{LaNiO}_{3}$ & Romboédrica & $\begin{array}{l}\text { Paramagnetismo de Pauli. } \mathrm{T}_{\mathrm{p}}=-1780^{\circ} \mathrm{K} \text {, conductividad } \\
\text { metálica. } \\
77 \leq \mathrm{T} \leq 575^{\circ} \mathrm{K}: \chi_{\mathrm{g}}=4.210^{-6} \mathrm{emu} / \mathrm{g} \text {, independiente de la } \\
\text { temperartura. }\end{array}$ \\
\hline $\mathrm{LaMnO}_{3}$ & $\begin{array}{l}\text { Ortorrómbica } \\
(\mathrm{c} / \sqrt{ } 2<\mathrm{a}<\mathrm{b}) \Rightarrow \\
\text { Romboédrica } \\
\text { (transición a } 752\end{array}$ & $\begin{array}{l}2<\mathrm{T}<1500^{\circ} \mathrm{K} \text { - Antiferromagnetismo, } \mathrm{T}_{\mathrm{N}}=130^{\circ} \mathrm{K} \\
\mathrm{T}_{\mathrm{p}}=50^{\circ} \mathrm{K} \text {. } \\
\text { Ferromagnetismo parasitario. }\end{array}$ \\
\hline
\end{tabular}

\section{3. Magnetorresistencia.}

El modelo anterior no tiene en cuenta, sin embargo, el fenómeno de la magnetorresistencia y es preciso aplicar algunas modificaciones para entenderlo, debido a que no contempla factores como, las interacciones intensas entre electrones y tampoco con los fonones $(1,6)$. El efecto magnetorresistivo(1, 5) consiste en la variación de la resistencia eléctrica al aplicarse un campo magnético. Se denomina gigante o colosal en función de la importancia de dicho fenómeno, en el primer caso el campo magnético rebaja la resistividad del material en torno a la mitad de la original mientras que en el segundo puede disminuirse en varios órdenes de magnitud. Ambos fenómenos se dan en algunos óxidos $\mathrm{ABO}_{3}$ como las manganitas donde han sido más estudiados, las cobaltatos son otra posibilidad interesante a revisar en este sentido en próximos trabajos. La MRC se observa en materiales cuya resistividad crece con la temperatura en el estado ferromagnético y, sin embargo, en el paramagnético se comporta como la de un 
semiconductor o un aislante, la transición metal asilante tiene lugar siempre en el entorno de $\mathrm{T}_{\mathrm{C}}$. Se describen a continuaciòn dos teorías explicativas del proceso que conectan con lo visto en el artículo previo de esta serie (1):

1. Polarones magnéticos: Para comprender su aparición es necesario tener en cuenta los importantes efectos de canje que ya se han descrito. Estas cuasipartículas se generan cuando un electrón de conducción se acopla a través de las interacciones de doble canje con los electrones $3 \mathrm{~d}$ o $4 \mathrm{f}$ de los cationes cercanos, de este modo estos últimos tienden a ordenar sus spines incluso en la zona paramagnética, como consecuencia el electrón de conducción queda atrapado en un potencial de canje generado por ese orden magnético. Se trata de una interacción a corto alcance y va acompañada por una deformación elástica del entorno, en el que queda localizado este electrón de conducción rodeado por un alineamiento de spines creado por él mismo, y que se desplaza con él, es el llamado polarón magnético. De Teresa e Ibarra (11) estudian los óxidos de $\mathrm{Mn}$ asegurando que para $\mathrm{T}<\mathrm{T}_{\mathrm{C}}$ la conducción en la fase ferromagnética es metálica y no existen polarones magnéticos, el único efecto de la aplicación de un campo magnético se debe a la fuerza de Lorentz que apenas modifica la resistividad. En cambio en el caso $T>T_{C}$ es donde surge el fenómeno de magnetorresistencia gigante o colosal, debido a la presencia de polarones en la fase paramagnética, ya que la aplicación de un campo magnético tiende a deslocalizar los electrones de conducción aumentando su movilidad y reduciendo la resistividad. El proceso que tiene lugar está restringido en torno a la temperatura de Curie, a la cual surge una transición espontánea aislante metal, o puede inducirse, a temperaturas próximas a ella, por aplicación de un campo $\mathrm{H}$ que provoca el crecimiento de los polarones, y cuando alcanza $1 \mathrm{~T}$ produce su fusión estableciéndose el orden ferromagnético en todo el material y la conducción metálica.

2. Desplazamiento del límite de movilidad: Basándose en el modelo s-d desarrollado por Kogan y Auslender (12), Bebenin y Ustinov $(13,14)$ proponen una sencilla teoría explicativa del proceso de la magnetorresistencia colosal para las manganitas, cuyo eje fundamental es el desplazamiento del límite de movilidad. Dado que la banda de valencia de estos óxidos es muy estrecha, y en ella se sitúa $\mathrm{E}_{\mathrm{F}^{\prime}}$ sus estados son muy sensibles a la presencia de desorden o distorsiones en la red, y estas sólo dependen de $\mathrm{T}$ si se deben a fluctuaciones magnéticas o vibraciones de red. La posición del límite de movilidad $\mathrm{E}_{\mathrm{C}}(1,6)$ para los huecos cambia con la temperatura, y se puede tomar como hipótesis, que la movilidad está controlada fundamentalmente por las oscilaciones magnéticas, entonces, conforme aumenta la temperatura lo hace el desorden y el límite de movilidad se desplaza a energías superiores, siendo extremadamente repentino el desplazamiento en el punto de transición magnética, debido a la gran cantidad de fluctuaciones magnéticas. Esto conlleva un gran cambio en el número de cargas, que se sitúan en estados localizados por la modificación de la posición relativa de $\mathrm{E}_{\mathrm{C}} \mathrm{y}_{\mathrm{F}}$ generando una transición metal aislante en $T_{C}$ (recordando siempre que hablamos de huecos). El estado paramagnético presentará estados localizados y una conducción de tipo de salto variable, mientras que en el ferromagnético estarán deslocalizados. Al aplicar un campo magnético $\mathrm{E}_{\mathrm{C}}$ se desplaza a energías inferiores, como consecuencia de la reducción del orden magnético. Este modelo demuestra que el campo también modifica la temperatura de transición siendo función lineal de $\mathrm{H}$.

\section{PROPIEDADES CATALÍTICAS}

El estudio de la conexión entre las propiedades electrocatalíticas de compuestos con metales de transición, y los parámetros electrónicos o composicionales es especialmente interesante en caso de las perovsquitas $\mathrm{ABO}_{3}$ donde sólo el catión $\mathrm{B}$ ( $3 \mathrm{~d}, 4 \mathrm{~d}, 5 \mathrm{~d})$ es catalíticamente activo y cuya valencia depende del A, permitiendo la estabilidad de estados mixtos de valencia (caso del $\mathrm{Co}, \mathrm{Mn}$...), pero conservando la estructura cristalina. Este conocimiento es básico para el diseño (no empírico) de composiciones con la selectividad y actividad requerida en aplicaciones tales como los dispositivos electroquímicos la conexión con todo lo anterior a través de los parámetros citados es fundamental en este sentido.

\subsection{Catálisis Heterogénea: Las perovsquitas de metales de transición.}

Un catalizador modifica la velocidad de las reacciones sin sufrir él un cambio químico permanente. Actúa activamente en las reacciones modificando su curso normal y reduciendo la entalpía de activación pero no altera el equilibrio. En el caso de la catálisis heterogénea en que el catalizador es de contacto, la superficie confina a los reactivos en un espacio bidimensional aumentando la probabilidad de colisión y reacción, allí se adsorben los reactivos en puntos favorables (centros activos).

En las perovsquitas los iones A son generalmente inactivos catalíticamente y los iones B se sitúan a distancias suficientemente grandes unos de otros (en torno a $4 \mathrm{~A}$ ), como para que las moléculas de los gases reactivos interaccionen con una única posición preferente. Incluso para un único centro activo de metal B, es posible modificar su estado de valencia y muchas propiedades físicas, seleccionando apropiadamente el catión A $(15,16)$. En un proceso catalítico superficial la reacción sucede por la secuencia de los siguientes pasos: adsorción (física o química), difusión superficial, mecanismo de reacción (formación de compuestos intermedios $<\mathrm{DH}_{\text {act' }}$ rotura y formación de enlaces, reestructuración molecular), desorción de productos (liberación de productos y recuperación de la estructura superficial del catalizador).

\section{1. 1. PARÁMETROS CONDICIONANTES DE LA ACTIVIDAD CATALÍTICA.}

Para relacionar la actividad catalítica de un compuesto con sus propiedades físicas, es preciso conocer detalladamente el mecanismo de la reacción catalizada, por ello se seleccionan reacciones de prueba y muestreo. Voorhoeve (17) establece una diferenciación entre dos clases de reacciones que le permite al mismo tiempo buscar la conexión de la actividad electrocatalítica en ambos casos con características físicas notablemente distintas:

1. Procesos catalíticos intrafaciales: Relacionados con parámetros termodinámicos como la entalpía de formación de vacantes de oxígeno (y el cambio de valencia de B). En este caso el catalizador actúa como reactivo, que es parcialmente consumido y regenerado en un ciclo continuo. Como es el caso de la reducción del NO $(17,18)$, que tiene lugar a través de la quimisorbción, en una posición de vacante de oxígeno. El número de estas vacantes en la superficie, y por tanto el parámetro físico de la energía de enlace, para el oxígeno en la superficie $\mathrm{E}(\mathrm{O})$, condiciona la eficacia del catalizador. Según 
la superficie elegida esta energía depende de un enlace u otro, en el caso de la superficie (100) puede modificarse a través de la sustitución de cationes en la posición A. La actividad catalítica crece en este caso conforme decrece el valor de $E(O)$, modificable a través de combinaciones de $\mathrm{A}_{\mathrm{x}} \mathrm{A}_{1-\mathrm{x}}^{\prime}$.

2. Procesos catalíticos suprafaciales: Relacionados con parámetros electrónicos. El catalizador se presenta como una superficie, que actúa como soporte y proveedor de orbitales de la energía y simetría apropiada, para que se dé el enlace con los reactivos y los productos intermedios. Es el caso de la oxidación del CO y la reducción del oxígeno a bajas temperaturas $\left(<200^{\circ} \mathrm{C}\right)(17)$. Será en estos últimos procesos, en los que se verá, con mayor interés, poder identificar, más fácilmente, en ellos los efectos del orden magnético en superficie, del campo cristalino o de la semiconducción. Existe una controversia notable en la literatura acerca de los modelos, que pueden explicar reacciones como las anteriores. Es posible extrapolar algunos de los modelos desarrollados, para los catalizadores metálicos superficiales al caso suprafacial suponiendo que las posiciones de adsorción preferente sean las de $\mathrm{B}$. El proceso catalítico está intensamente influenciado por la etapa de la adsorción (quimisorbción), en que se produce una transferencia de carga y redistribución de los estados electrónicos entre adsorbato y superficie generando enlaces. Los orbitales enlazantes y antienlazantes de la molécula del gas reactivo interaccionan, con los electrones deslocalizados (s y p) del metal generando desplazamientos en energías y ensanchamientos de los OM en resonancias, además se produce la interacción con los estados más localizados de la banda d del metal de transición, siendo estos electrones los que dan el carácter catalítico, se genera así la hibridación de los estados d citados con los nuevos estados resonantes configurándose una molécula superficial (19) con nuevos estados enlazantes y antienlazantes. La fuerza de este enlace condiciona la actividad catalítica del sistema permitiendo o no, la disociación de las moléculas de reactivo y la posterior desorción. En este modelo existen una serie de parámetros que limitan la reactividad de la superficie, y que están íntimamente relacionados con la interacción entre los $\mathrm{OM}$ y la banda d. Se trata de la posición relativa en energías de los estados enlazantes y antienlazantes del reactivo, (siendo estos niveles resonantes), frente a la banda d, el grado de ocupación de los estados antienlazantes de la molécula superficial y asociado a $\mathrm{E}_{\mathrm{F}}$ (grado de ocupación de los estados d del metal) y a la función de trabajo de la superficie (no hay que olvidar que las bandas allí se estrechan), y el grado de solapamiento entre los estados d y las resonancias de los $\mathrm{OM}\left(\mathrm{V}_{\text {ads,fik }}(20,21)\right.$ elemento de la matriz de energía de interacción relacionada con la integral de canje entre la fdo de adsorbato y el estado $\mathrm{K}$ de la banda del metal). La estructura de bandas de estos óxidos $(1,2)$, estaría relacionada de modo directo con la actividad electrocatalítica en el caso de los procesos suprafaciales y también con los intrafaciales, ya que la fuerza del enlace con los oxígenos y por tanto $\mathrm{E}(\mathrm{O})$, modifica esta estructura a través de la $\mathrm{D}_{\text {cac }}$ (1). Así mismo el carácter localizado o colectivo de sus electrones, establecería diferencias de comportamiento en este sentido. La energía y simetría de los orbitales implicados en el proceso, se vería contemplada en el término de interacción energética entre estados electrónicos del reactivo y del óxido, a través de la integral de solapamiento y de la de canje. El tratar con compuestos de banda estrecha puede permitir que los estados de la perovsquita, presenten un solapamiento notable con la distribución de carga del adsorbato, de modo que en un intervalo de energías muy estrecho el término $V_{\text {ads,fik }>}$ tiene un valor muy alto.

\section{1. 2. REACCIONES CONCRETAS: REDUCCIÓN DEL O $\mathrm{O}_{2}$. OXIDACIÓN DEL CO}

Se trata como ejemplo el caso de la reducción del $\mathbf{O}_{2}$, que es especialmente polémico. Brenet (22) presenta una relación entre la presencia de parejas de estados $\mathrm{B}^{4+} / \mathrm{B}^{3+}$ y la reactividad de los óxidos. Staikos (23) explica la actividad reductora del $\mathrm{O}_{2}$ de las perovsquitas, a través de parámetros cristalográficos y termodinámicos. Manoharan y Shukla (24) proponen un mecanismo de reacción en que las vacantes de oxígeno juegan el papel fundamental, a través de la no estequiometría del óxido. Matsumoto et al. (25) presentan la posibilidad de explicar la actividad catalítica de los óxidos de tipo perovsquita frente a esta reacción concreta, a través de la estructura de bandas propuesta por Goodenough $(1,2)$. Los últimos autores estudiaron la reducción del oxígeno en $\mathrm{LaNiO}_{3}$ y óxidos relacionados con ella $(26,27)$, para luego extender sus conclusiones sobre la influencia de la naturaleza de la banda de conducción, en la actividad catalítica a todos los óxidos tipo perovsquita de metales de transición en esa reacción específica. De acuerdo con Goodenough(2), existen tres tipos de bandas de carácter $\mathrm{d}$ en los óxidos de metales de transición: la p* generada por la interacción entre orbitales $t_{2 g}$ de B-B, la p formada por la interacción entre un orbital $t_{2 g}$ de $B$ y uno $p_{p}$ del oxígeno en un enlace $\mathrm{B}-\mathrm{O}-\mathrm{B}, \mathrm{y}$ una banda $\mathrm{s}^{*}$ a través de un orbital $\mathrm{e}_{\mathrm{g}}$ de $\mathrm{B}$ y otro $\mathrm{sp}_{\mathrm{s}}$ del oxígeno en B-O-B. Existen dos modos posibles para la adsorción de la molécula de oxígeno (26) sobre la superficie del catalizador. La adsorción del tipo side-on (sobre una cara), parece ser improbable ya que el $\mathrm{O}_{2}$ se adsorbería de modo que el ión del metal de transición quedaría coordinado octaédricamente. En este caso la longitud de enlace, entre los dos átomos de la molécula $\mathrm{O}_{2}$, debería ser muy elevada, para adaptarse a la estructura cristalina. La otra posibilidad es la adsorción de tipo end-on (al final), que es la más probable. La molécula de oxígeno se adsorbe sobre las posiciones activas superficiales, que resultan ser las de los cationes $\mathrm{B}$. En este caso un orbital de la molécula $\mathrm{O}_{2}$ se orienta hacia un orbital e del metal de transición. La probabilidad de solapamiento entre el orbital molecular del adsorbato, y el $\mathrm{e}_{\mathrm{g}}$ depende del valor de la integral de solapamiento $\mathrm{D}_{\text {cac }}^{\mathrm{s}}$. Los parámetros, que afectan fundamentalmente a este proceso, serán la densidad de estados electrónicos en torno al nivel de Fermi, y la integral de canje J entre funciones de onda del reactivo y el catalizador. Experimentalmente $(26,27)$ se deduce, que es el factor J el determinante de la actividad catalítica de la perovsquita, en este caso, y al ser proporcional a la integral de solapamiento (Ec.7), entre las fdo de electrones del centro activo $\mathrm{B}$ y del átomo de óxigeno adyacente del compuesto intermedio $\left(\mathrm{O}_{2} \mathrm{H}\right)$ debe existir una conexión con la integral de solapamiento $\mathrm{D}_{\text {cac }}^{\mathrm{s}}$ del cristal. Entonces, si se asume, que la adsorción tipo end-on es la que tiene lugar, el solapamiento del orbital molecular $\mathrm{p}^{*}$ del $\mathrm{O}_{2}$, que está semiocupado, y presenta las máximas energías, con el orbital $t_{2 g}$ de B será muy débil. Es decir, los electrones de la banda s* serán transferidos más facilmente al orbital molecular $\mathrm{p}^{*}$ del oxígeno, que los de la banda p*. Esta es una razón que puede explicar la actividad catalítica en la reducción del oxígeno para los óxidos de metales de transición con banda $\mathrm{s}^{*}$ conteniendo electrones. El caso de la oxidación del CO es también polémico. Voorhoeve $(17,18)$ lo estudió como reacción de prueba sobre la familia $\left(\mathrm{La}, \mathrm{A}^{\prime}\right) \mathrm{CoO}_{3}$ donde $\mathrm{A}^{\prime}$ fue elegido para variar la valencia del Co (por el fenómeno de compensación de la carga), observándose que la introducción de $\mathrm{Co}^{2+}$ daba lugar al incremento de la actividad catalítica mientras que el $\mathrm{Co}^{4+}$ la 
reducía. La actividad en la oxidación del CO se relacionó con el grado de ocupación de los niveles d en superficie (estado electrónico de B, (figura 1).

La actividad es máxima para la configuración $\mathrm{e}_{\mathrm{g}}^{\mathrm{d}} \operatorname{con} 0<\mathrm{d}<1$ y decae al crecer esta ocupación o cuando los orbitales $t_{2 g}$ contienen menos de tres electrones. Los datos cinéticos permiten demostrar que el enlace del CO en superficie con el catión B como carbonilo (17) es una parte crítica del proceso. Se produce la donación del par de electrones del carbono a un orbital $\mathrm{d}_{\mathrm{z}}^{2}$ vacío, generando un enlace $s$ junto a una transferencia de electrones $t_{2 g}$ del metal al orbital molecular $\mathrm{p}^{*}$ del CO. Por ello es favorable que los estados e estén vacíos y los $\mathrm{t}_{2 \mathrm{~g}}$ llenos, y para un ión metálico en superficie (100). el orbital $\mathrm{d}_{\mathrm{z}}^{2}$ es el fundamental de los $e_{g}$ por lo que, para tenerlo al menos parcialmente vacío es preciso que la ocupación de todos los $\mathrm{e}_{\mathrm{g}}^{\mathrm{d}}$ sea baja $(0<\mathrm{d}<1)$. Tascón, Fierro y González Tejuca (28) estudiaron la oxidación del CO sobre la familia $\mathrm{LaBO}_{3}$ obteniendo máximos en la actividad para el $\mathrm{Mn}^{3+}\left(\mathrm{d}^{4}\right)$ y el $\mathrm{Co}^{3+}\left(\mathrm{d}^{6}\right)$ confirmando así la relación entre la simetría cristalina local de los cationes superficiales, con la quimisorbción y la catálisis a través de la teoría del campo cristalino (29). También aparecen estos dos máximos de actividad en ciertas reacciones, que involucran hidrógeno así como en los cálculos teóricos del incremento de la energía del campo cristalino, debido al cambio de estado de coordinación en la superficie. Así se puede relacionar la configuración electrónica del catión del metal de transición, y las interacciones localizadas con la actividad catalítica a través del entorno cristalino. En general se puede afirmar, que para la oxidación del CO la actividad crece cuando el nivel de Fermi se traslada hacia el centro del nivel $e_{g}^{*}$ de menor energía. Si la banda e está vacía o completamente llena la actividad se reduce. En la superficie (100), este nivel es el $d_{z 2}$ antienlazante y puede ser este el nivel de energía y simetría adecuado para que se dé el solapamiento idóneo para acelerar el paso determinante de la cinética del proceso de oxidación del CO.

\section{CONCLUSIONES}

En el presente trabajo se ha hecho una revisión de los modelos de la Física y Química del estado sólido que dan una explicación de las propiedades magnéticas y electrocatalíticas de los óxidos tipo perovsquita con metales de transición del primer período. Se ha pretendido establecer una relación entre todos ellos de modo que exista una visión integradora de los parámetros, que pueden condicionar el comportamiento de estos sistemas. Este trabajo de revisión y unificación completa con una primera parte que estudia las propiedades de conducción electrónica dando así una clara visión global de las características de esta serie de compuestos isoestructurales tan interesantes para la ciencia de materiales.

\section{BIBLIOGRAFÍA}

1. L. Sánchez, J.R. Jurado “Correlación entre la estructura de bandas y las propiedades físicas de óxidos cerámicos de estructura perovsquita con metales de transición: (I) Conducción Electrónica", enviado al Boletín de la Sociedad Española de Cerámica y Vidrio, 1999.

2 . J.B.Goodenough. "Metallic Oxides", Progress in Solid State Chemistry vol 5, Ed. H. Reiss. Pergamon Press, NY 1971, 145-399.

3. J.B.Goodenough, J.M.Longo "Crystallograpihc and magnetic properties of perovskite and perovskite related compounds" Landolt Börnstein New Series, K.H.Hellwege, A.M. Hellwege, vo4, part a, 126. Springer-Verlag, Berlin, 1970.

4. N.W.Ashcroft, N.D. Mermin, “Solid State Physics", CBS publishing Asia LTD., 1988.

5. B.D. Cullity "Ihntroduction to Magnetic materials", Addison Wesley series in Metallury and Materials, Addison Wesley, 1972.

6. N. Tsuda, K. Nasu, A. Yanase, K. Siratori, "Electronic Conduction in Oxides",

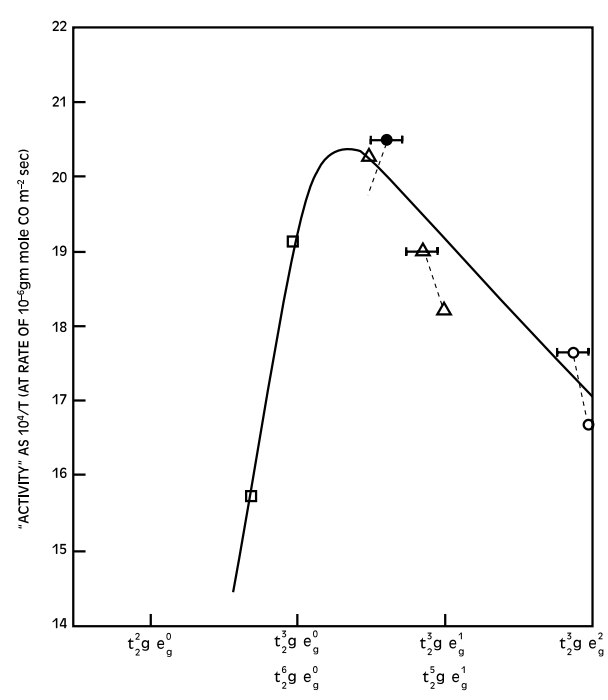

Fig. I. Actividad de las perovsquitas en la oxidación del $\mathrm{CO}$ vs la configuración electrónica del catión $\mathrm{B}: \mathrm{ACrO}_{3}, \Delta \mathrm{AMnO}_{3}, \mathrm{O} \mathrm{AFeO}_{3}$, - $\mathrm{ACoO}_{3}$. Se expresa la actividad como $10^{4} / \mathrm{T}$ siendo $\mathrm{T}$ la temperatura a la que el ritmo de oxidación es $10^{-6} \mathrm{gm} \mathrm{mol} \mathrm{m}^{-2} \mathrm{sg}$ en una mezcla 2:1 de $\mathrm{CO}_{\text {y }} \mathrm{O}_{2}$ a la presión atmosférica (según Voorhoeve (17)).

Springer Series in Solid State Sciences, springer Verlag, Berlin, 1991.

7.J.M. Ziman "Electrons and phonons. The Theory of Transport Phenomena in Solids", Oxford Univ. Press, London 1960.

8.K.W.H.Stevens, “Spin Hamiltonians", Ed.G.T.Rado, H. Suhl, “Magnetism”, vol 1, 1, 1969.

9. J.B.Goodenough "Magnetism and Crystal structure in Nonmetals", Ed.G.T.Rado, H. Suhl, "Magnetism", vol 2, 1, 1969.

10. F.S. Galasso "Structure, properties and preparation of perovskite type compounds", Pergamon, Oxford, 1969.

11. J.M. De Teresa, M.R. Ibarra “Polarones magnéticos", Investigación y Ciencia, abril 1998.

12. E.M. Kogan, M.I. Auslender, Phys. Stat. Sol.(b), 147, 1988.

13. N.G.Bebenin, V.V.Ustinov "Conduction and Disorder in $\mathrm{LaMnO}_{3}$-based Materials", to be published in J. Phys Cond. Matter. 1999.

14. N.G. Bebenin et al, "Charge Carriers in $\mathrm{La}_{0.67 .} \times \mathrm{YxBa}_{0.33} \mathrm{MnO}_{3}$ ", Solid State Comm., vol 00, 0, 1998.

15. L.G.Tejuca, J.L.G. Fierro, J.M.D.Tascón "Structure and Reactivity of Perovskite type oxides", Adv. Cat. Vol 36, 1989

16. J. Happel, L. Bajars, M. Hnarow, "Catalytic properties of the perovskites", Base metal oxide Catalyst, Dekker, N.Y., 1977.

17. R.J.H.Voorhoeve "Perovskite related oxides as oxidation-reduction catalysts", Materials Science Series, Edts. J.J.Burton, R.L.Garten, Academic Press, N.Y. 1977.

18. R.J.H.Voorhoeve, D.W.Johnson Jr, J. P.Remeika, P. K. Gallagher, “Perovskite Oxides: Materials Science in Catalysis", Science, vol 195, 4281, 1977.

19. G. A. Somorjai "Introduction to Surface chemistry and catalysis", John Wiley \& Sons, USA, 1994.

20. B. Hammer, J.K. Norrskov "Electronic factors determining the reactivity of metal surfaces", Surf. Sci., 343, 211-220, 1995.

21. A. Zangwill "Physics at Surfaces", Cambridge University Press, 1992.

22. J. P. Brenet, J. Power Sources 4, 183, 1979.

23. D. N. Staikos, ECS Estended Abstracts 2, 1644, 1979.

24. R. Manoharan, A. K. Shukla, “ Oxides supported carbon air-electrodes for alkaline solution power devces", Electrochimica Acta vol 30, 2, pp 205-209, 1985.

25. Y. Matsumoto, H. Yoneyama, H. Tamura, “ The Mecahnism of oxygen reduction at a $\mathrm{LaNiO}_{3}$ electrode", Bull. Chem. Soc. Jap., vol 51, 7, 1978.

26. Y. Matsumoto, H. Yoneyama, H. Tamura, "Catalytic Activity for electrochemical reduction of oxygen of lanthanum nickel Oxide and related oxides"J. Electroanal. Chem, 79, 1977.

27. Y. Matsumoto, H. Yoneyama, H. Tamura, "Influenceof the nature of the conduction band of transition metal oxides on catalytic activity for oxygen reduction", J. Elechtroanal. Chem. 83, 237, 1977.

28. J. M. D. Tascón, L. González Tejuca, “ Catalytic Activity of perovskite-type oxides $\mathrm{LaMeO}_{3}$ ", React. Kinet. Catal. Lett., Vol 15, 2, 1980

29. D.A. Dowden, D. Wells, " A Crystal field interpretation of some activity patterns", Actes 2e Congr. Int. Catalyse ed Technip, Paris, vol2, p 1499, 1961.

30. S. Nomura "Crystallographic and magnetic properties of perovskite and perovskite related compounds", Landolt Börnstein New Series, K.H.Hellwege, A.M. Hellwege, vol 12, part a, p 368. Springer-Verlag, Berlin, 1978.

Recibido: 01.05.99

Aceptado: 12.08 .00 